\title{
SPICE Modelling of Photoluminescence and Electroluminescence Based Current-Voltage Curves of Solar Cells for Concentration Applications
}

\author{
D. Alonso-Álvarez* and N. Ekins-Daukes \\ Imperial College London, London, United Kingdom \\ *Corresponding Author: d.alonso-alvarez@imperial.ac.uk
}

Received 5 November 2015; Accepted 3 June 2016;

Publication 1 August 2016

\begin{abstract}
Quantitative photoluminescence (PL) or electroluminescence (EL) experiments can be used to probe fast and in a non-destructive way the currentvoltage (IV) characteristics of individual subcells in a multi-junction device, information that is, otherwise, not available. PL-based IV has the advantage that it is contactless and can be performed even in partly finished devices, allowing for an early diagnosis of the expected performance of the solar cells in the production environment. In this work we simulate the PL- and ELbased IV curves of single junction solar cells to assess their validity compared with the true IV curve and identify injection regimes where artefacts might appear due to the limited in-plane carrier transport in the solar cell layers. We model the whole photovoltaic device as a network of sub-circuits, each of them describing the solar cell behaviour using the two diode model. The subcircuits are connected to the neighbouring ones with a resistor, representing the in-plane transport in the cell. The resulting circuit, involving several thousand sub-circuits, is solved using SPICE.
\end{abstract}

Keywords: Semiconductors, multi-junction solar cells, photoluminescence, SPICE.

Journal of Green Engineering, Vol. 5, 33-48.

doi: 10.13052/jge 1904-4720.5343

(C) 2016 River Publishers. All rights reserved. 


\section{Introduction}

Electroluminescence and photoluminescence spectroscopy and imaging have been extensively used for the characterisation of solar cells and modules in recent years, specially in the silicon industry [1-5]. They allow for spatially resolved identification of shunts, inhomogeneity of the materials, the influence of grain boundaries, the quality of the metallisation or minority carrier lifetimes. Recent works have extended the techniques to multi-junction (MJ) solar cells, where some degree of spectral resolution, rather the spatial resolution, is necessary in order to distinguish the luminescence from the different subcells [6-11].

In a previous work, we used photoluminescence spectroscopy as a contactless, fast method to evaluate the current-voltage (IV) characteristics of each junction in MJ devices, estimating the solar cell characteristics such as open circuit voltage $\left(\mathrm{V}_{\text {oc }}\right)$ or fill factor (FF) [12]. Such contactless technique allows for a fast screening of devices in the industry environment as well as tool for early diagnosis of the quality of the epitaxial process, given that a full device structure is not necessary. We found that, although the technique provided reliable results in $\mathrm{MJ}$ structures with up to 6 junctions when compared to the better known electroluminescence method, the current tended to be overestimated, specially at lower injection levels. We attributed that behaviour to the lateral transport that takes place in the solar cell layers whenever carrier injection (either electrical or optical) is not homogeneous in the whole sample.

In this work, we model the experimental PL- and EL-based IV curves presented in Ref [12] in the presence of inhomogeneous current injection using a distributed electrical circuit that can be solved using SPICE.

\section{Modelling the Solar Cell with SPICE}

\subsection{The Electrical Model}

We use a 3D electrical network to model the flow of injected current through the solar cell. This type of formalism is widely used to simulate the performance of solar cells when the effect of a spatial variable need to be incorporated in the model. Such variable can be the design of the front metal grid, in order to minimise the effect of series resistances [13], the inhomogeneous illumination profile in concentrator devices and the impact of such inhomogeneity into the transport through the tunnel junctions $[14,15]$, or the distribution of defects or inhomogeneities [16-18]. 
The device area is divided into individual units, each of them consisting into a stack of diodes and resistors that connect each unit to their neighbours. Depending on the location of the unit, the structure of the equivalent electrical circuit is different. Figures 1 and 2 illustrates this formalism. Two regions are distinguished: the metal and the aperture. In the aperture, between the metal fingers, the solar cell is modelled using two diodes with reversed saturation

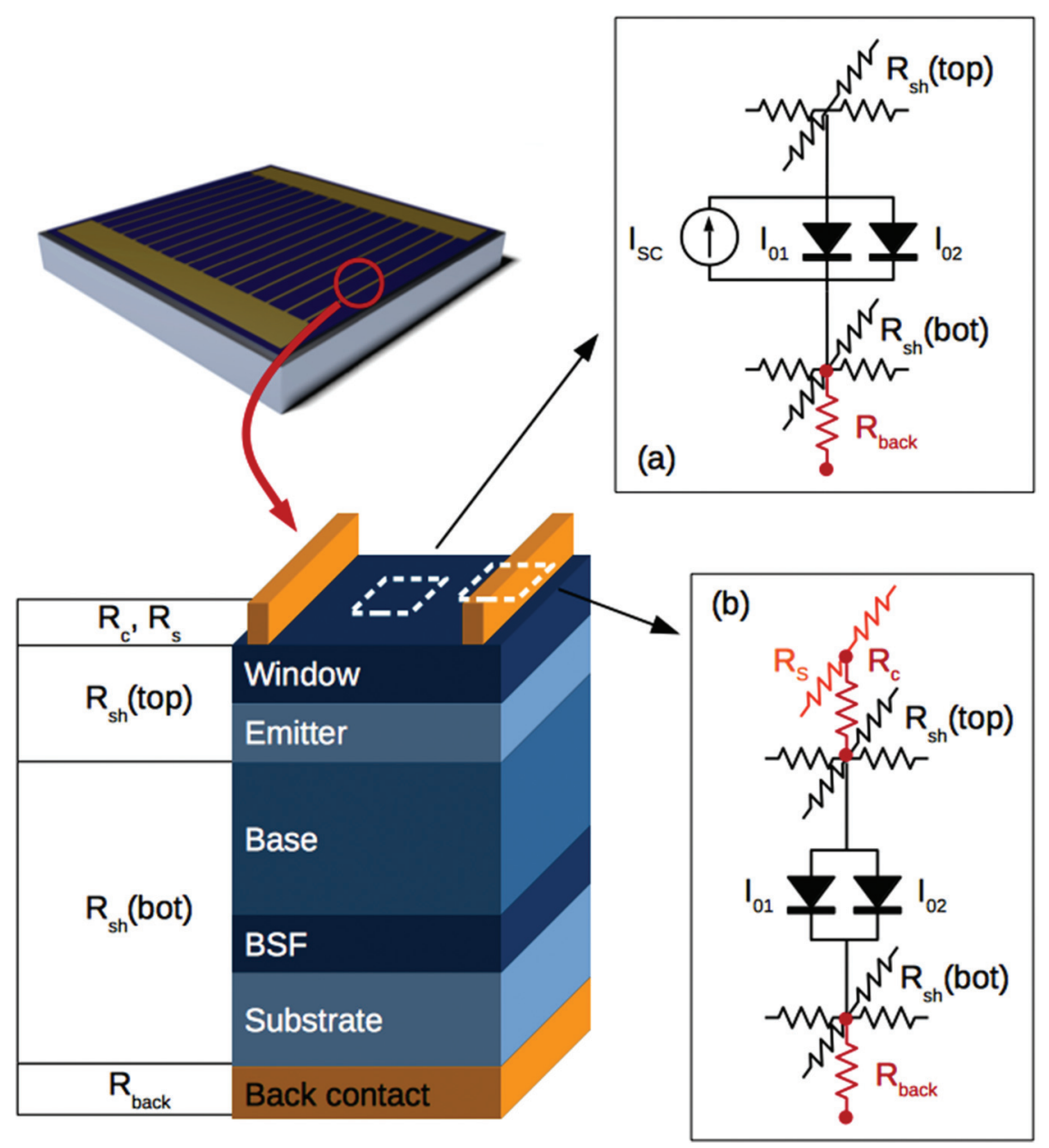

Figure 1 SPICE model for the 1-junction solar cell in the vertical direction. (a) Equivalent circuit between the metal fingers, in the aperture region. (b) Equivalent circuit in the region covered with metal. 


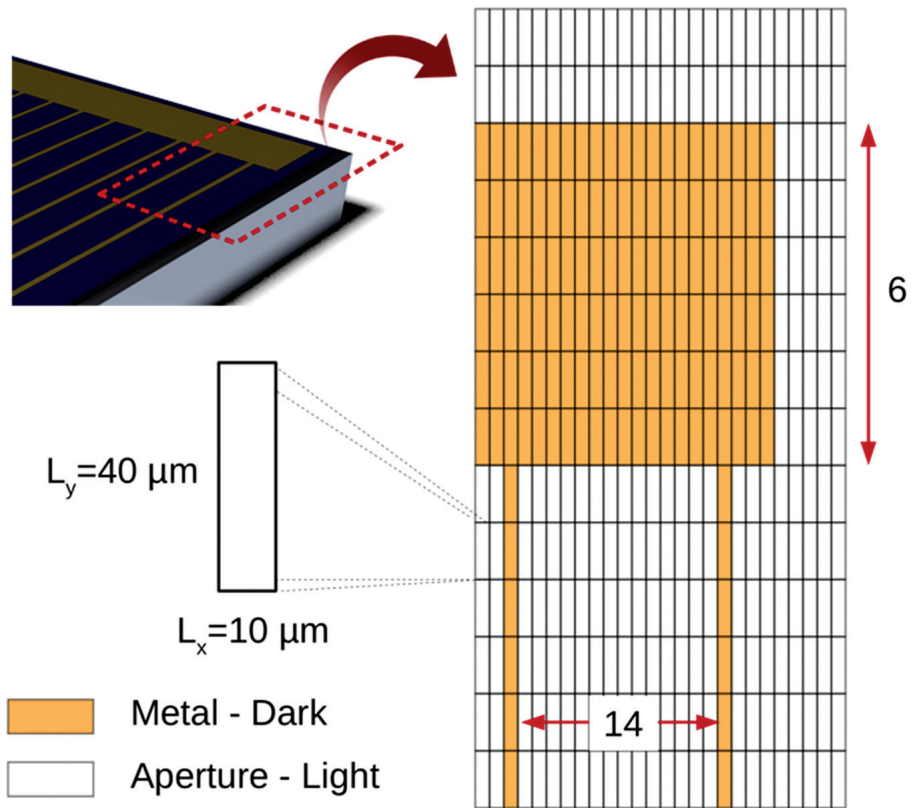

Figure 2 SPICE model for the 1-junction solar cell in the plane. The whole mesa is divided into small, asymmetric units $10 \times 40 \mu \mathrm{m}$ which are interconnected with the resistors shown in Figure 1, allowing current to flow laterally across the structure.

currents $\mathrm{I}_{01}$ and $\mathrm{I}_{02}$ and ideality factors 1 and 2, respectively, and with the photogeneration included as a current source $\left(\mathrm{I}_{\mathrm{sc}}\right)$ connected in parallel with the diodes. Sheet resistances above and below the junction, $\mathrm{R}_{\mathrm{sh}}(\mathrm{top})$ and $\mathrm{R}_{\mathrm{sh}}$ (bot), account for the lateral transport. We will describe the origin of these resistances later. Beneath the metal, there is no current source, as the region is in the dark, and there are extra resistances accounting for the contact between the metal and the semiconductor $\left(\mathrm{R}_{\mathrm{c}}\right)$ and the transport along the metal finger $\left(\mathrm{R}_{\mathrm{S}}\right)$. Steiner et al. considers a third type of unit, those next to the perimeter of the cell where higher recombination is to be expected [13]. In this work we neglect that effect for simplicity. The resulting network is solved using NGSPICE [19].

In order to maximise the calculation speed, we consider a homogeneous mesh of rectangular units with sizes $\mathrm{L}_{\mathrm{x}}=10 \mu \mathrm{m}$ and $\mathrm{L}_{\mathrm{y}}=40 \mu \mathrm{m}$ (Figure 2). This makes each metal finger one unit wide and the aperture between fingers 14 units wide. Considering the symmetry of the samples and the collection and excitation spots, only half of the device needs to be simulated, further reducing 
the computation time. Inhomogeneous meshes to increase the resolution of specific regions of the device are also possible [18].

\subsection{Calculation of the Resistance}

The resistances of the circuit shown in Figure 1 can be defined in terms of the resistivities, the sheet resistances of the semiconductor layers and the geometry of the units as described in Ref. [13]:

$$
\begin{aligned}
R_{s h}^{x} & =\frac{L_{x}}{L_{y}} R_{s h}, \quad R_{s h}^{y}=\frac{L_{y}}{L_{x}} R_{s h} \\
R_{s}^{x} & =\frac{L_{x}}{h L_{y}} \rho_{m}, \quad R_{s}^{y}=\frac{L_{y}}{h L_{x}} \rho_{m} \\
R_{c} & =R_{\text {back }}=\frac{1}{L_{x} L_{y}} \rho_{c}
\end{aligned}
$$

where Equation 1 applies to both top and bot sheet resistances in Figure 1, $\rho_{\mathrm{m}}=2 \times 10^{-6} \Omega \mathrm{cm}$ is the linear resistivity of the metal fingers, $\rho_{\mathrm{c}}=3 \times 10^{-6}$ $\Omega \mathrm{cm}^{2}$ the resistivity of the metal-semiconductor interface, assumed equal for the front and back contact and $h$ is the height of the metal fingers, set equal to $2.2 \mu \mathrm{m}$. The superindex $x$ and $y$ indicate the direction of the resistance. These resistivities have been taken from [15] as representative values for these magnitudes and might be different in our devices.

The sheet resistance $R_{\mathrm{sh}}$ of a stack of several semiconductor layers is equal to the combination in parallel of the individual sheet resistances. For example, for $R_{\mathrm{sh}}(t o p)$ in Figure 1:

$$
\frac{1}{R_{s h}(\text { top })}=\frac{1}{R_{s h}(\text { window })}+\frac{1}{R_{s h}(\text { emitter })}
$$

Each of these can in turn be estimated from the thickness of the layer $d$, the majority carrier mobility $\mu$ and the doping $N$ [15]:

$$
\frac{1}{R_{s h}}=q d \mu N
$$

For single junction devices, further approximations can be done. A doped substrate has a very low sheet resistance consequence to its large thickness $(\sim 300 \mu \mathrm{m})$. As a result, Equation 4 calculated for the bottom layers can be approximated as $\mathrm{R}_{\mathrm{sh}}(\mathrm{bot}) \sim 0 \Omega / \square$. In this situation, the contact resistance at the back contact, $R_{\text {back }}$ can also be set to zero as the total contact area is the whole mesa. 


\subsection{Calculation of the Currents and Voltages}

In the simulation, we will give as input parameter the current injected in the device and get, as the output, the voltages across the diodes of the different circuit units. In the experiment, these voltages are obtained from the luminescence measurements, as discussed in Ref. [12].

For electrical injection, the current $I_{\mathrm{el}}$ is injected in the units forming the bus bars (larger metal pads in Figure 3a). For the optical injection, the current is injected in the area illuminated by the laser (Figure 3b). The $I_{\mathrm{sc}}$ of the sub-circuits corresponding to aperture units outside the illuminated area are set equal to zero. For the calculation of the IV curves based on the SPICE simulations and their comparison with the experiments, we make the same assumptions than in the latter:

- For EL, the recombination current density $J_{\text {rec }}$ is given by the electrically injected current $I_{\mathrm{el}}$ divided by the area of the device, defined by an etched mesa, $A_{\text {mesa }}=0.0547 \mathrm{~cm}^{2}$.

- For PL, $J_{\text {rec }}$ is the optically injected current $I_{\mathrm{op}}$ divided by the excitation area $A_{\text {ex }}=0.0141 \mathrm{~cm}^{2}$ :

It should be noted that the current in the case of electrical injection would be the same for all junctions in a MJ device, whereas the current in the optical injection is junction dependent. In order to simplify the comparison of the results, we will assume that $I_{\mathrm{op}}=I_{\mathrm{ex}}$ such that the EL- and PL-based IV curves are evaluated in the same current range.

The reverse saturation currents of the diodes $\mathrm{I}_{01}$ and $\mathrm{I}_{02}$ are taken from a fit of the experimental EL-based IV curves to a 2-diode model. As we will see

(a) Sample mesa $2270 \times 2400 \mu \mathrm{m}$

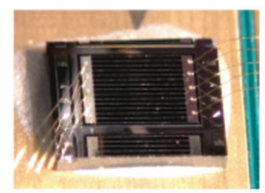

(b) Excitation spot $1200 \times 1500 \mu \mathrm{m}$

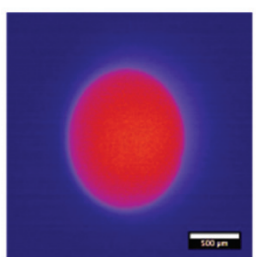

(c) Collection spot $\varnothing 650 \mu \mathrm{m}$

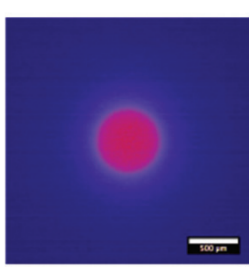

(d)

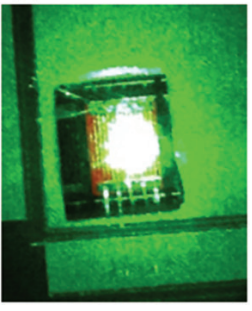

Figure 3 (a) Device under test, showing the pads connected with gold wires. (b) Laser spot, centred in the device mesa. (c) Collection spot, centred on the excitation spot. (d) The device illuminated with green laser light. The central spot of the laser is surrounded by red photoluminescence emitted by GaInP. 
in the next section, for simulation purposes and to illustrate the effect of lateral currents in the measured PL-based IV, this is a good approach. However, as the EL-based IV can also be affected by the lateral currents under high injection or high sheet resistance conditions, using these values for fitting the PL-based IV should be done with care.

For the calculation of the voltages needed to evaluate the IV curve, we will average the voltage drop across the diodes of all units in the collection area (Figure 3c). This is different that in the usual IV curves, where the voltage is given by its value in the bus bars (excluding the effect of series resistance in the wires and cables), but it is the voltage that is actually probed when using luminescence methods. The excitation and the collection areas are centred in the device mesa, meaning that the same region is probed in PL and EL experiments. The local recombination current density in each unit is the total current flowing for the two diodes of that unit.

\section{Results}

\subsection{Dependence of the Apparent IV Curve with $\mathbf{R}_{\mathbf{s h}}$}

Figure 4 shows the simulated results for the case of a GaInP solar cell. The colour maps on the top of the figure show the local voltage in the case of electrical injection (upper, EL) and optical injection (lower, PL) when $\mathrm{R}_{\mathrm{sh}}$ (top) $=1000 \Omega / \square$ is used. It can be seen that at lower injection levels, local voltage is homogeneous in the whole device. In other words, the injected current spreads easily in the lateral direction, biasing the entire mesa with the same voltage. As injection is increased, the effect of the limited sheet resistance becomes apparent, being clear from $100 \mathrm{~mA}$ that the voltage is no longer homogeneously distributed in the device: for the electrical injection, voltage is higher just beneath the metal fingers whereas for the optical injection this happens just in the excitation spot.

Based on these calculations, we can evaluate the PL- and EL-based IV curves based on the steps discussed in Section 2.3 for different sheet resistances (Figure 4, bottom). The true IV curve, based on the two diode model described above, is also shown. At lower injection levels, the EL-based IV curves follow closely the true IV curve, being the small difference consequence of the inplane mesh size described in Figure 2. At higher injection, the EL-based curves diverge due to the limited in-plane transport. The PL-based IV curves are systematically above the EL-based ones regardless of the sheet resistance. 

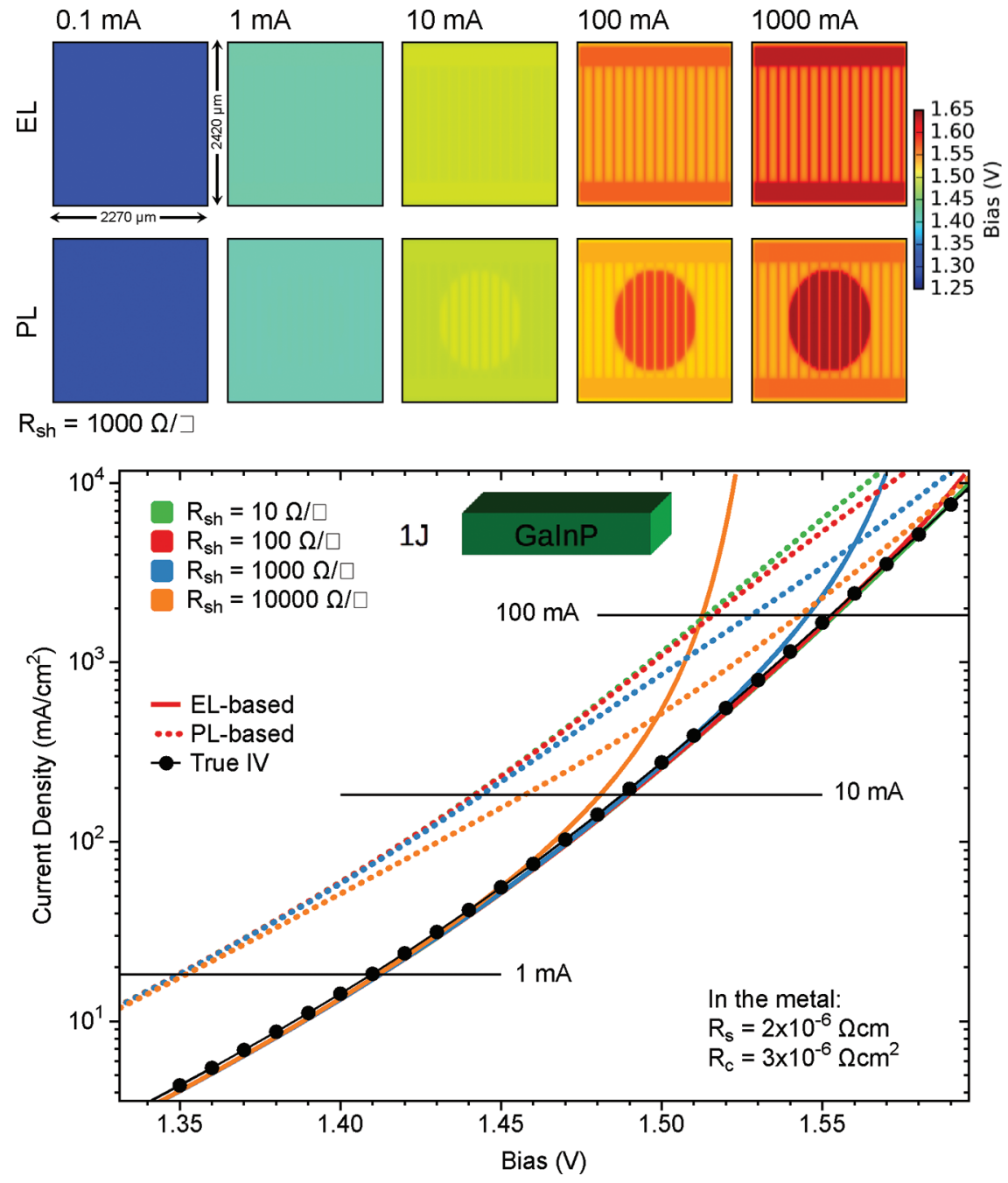

Figure 4 (Top) Color maps showing the local voltage for different levels of injected current in the case of electrical injection (EL) and optical injection (PL) when $\mathrm{R}_{\mathrm{sh}}(\mathrm{top})=1000 \Omega / \square$. (Bottom) Simulated EL- and PL-based IV curves for a GaInP solar cells with different values of $\mathrm{R}_{\mathrm{sh}}$ (top).

The ratio between both is 4.05 at $1.35 \mathrm{~V}$, which is almost identical to the ratio between the area of the mesa and the area of the laser spot $A_{\text {mesa }} / A_{\mathrm{ex}}=3.9$. As injection is increased, both sets of curves get closer, reaching the limit of crossing in the case of very high sheet resistances. 
This behaviour is consistent with the incorrect assumption made in the experiments that in the case of optical injection, recombination takes place just in the excitation region and in the electrical injection, recombination is always homogeneous in the whole mesa: the actual recombination region depends on the injection level and the sheet resistance.

This uncertainty in the recombination area plays a key role in the interpretation of the EL- and PL-based IV curves and could be exploited as a means of gathering information on the sheet resistances of buried junctions in multi-junction solar cells.

\subsection{Fitting of PL-based IV Curves}

Figure 5 shows the experimental and simulated PL- and EL-based IV curves for two single junction devices made of GaAs and GaInP. The sheet resistances that give the best match to the experimental data are indicated in Table 1. In both cases, it can be seen that it is possible to correctly reproduce both curves using the same set of parameters, namely the reverse saturation currents $\mathrm{I}_{01}$ and $\mathrm{I}_{02}$, and the sheet resistance for the top layers, supporting the validity of the modelling process with SPICE.

In general, the fitting of the PL-based IV at lower injection is poorer that at higher injection. This is consequence of the way voltages are determined in the experiment: the internal bias of the solar cell is calculated from the luminescence signal, which is weaker and noisier at lower injection levels, and therefore more influenced by random fluctuations of the background in the spectrometer. This effect is included in the larger error bars of the experimental data at lower injections, but might also result in a systematic overestimation of the voltage in that range.

A comparison can be made of the obtained sheet resistances and the nominal values calculated according to Equations 4 and 5. Nominal values for the thickness and the doping are used. We estimate the mobility from the composition of the layers following the method described in Ref. [20]. In both cases, the estimation of the sheet resistance is within the same order than the calculated one, although the exact values are not correctly reproduced. Part of this discrepancy can be related to the uncertainty of the experimental data and in the chosen values for the $\mathrm{I}_{01}$ and $\mathrm{I}_{02}$, as it has been discussed. Specially the first one, associated with radiative recombination and dominant at higher injection regimes must be taken with caution. However, another part of the discrepancy can be in the actual nominal values used in Equation 5. While the 

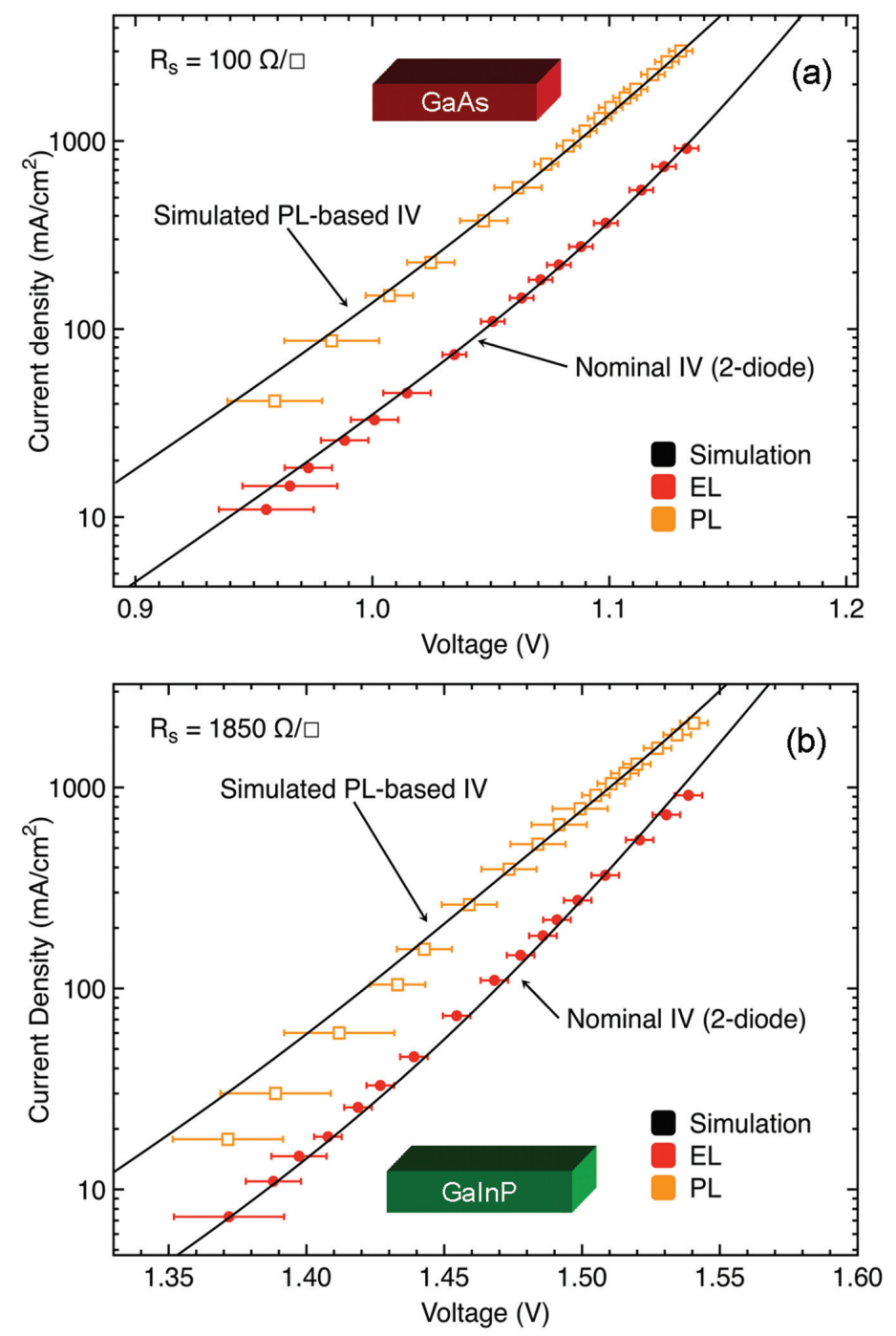

Figure 5 Experimental and simulated PL- and EL-based IV curves for (a) GaAs and (b) GaInP single junction solar cells.

Table 1 Modelled and nominal sheet resistances for the GaAs and GaInP solar cells

\begin{tabular}{lcc}
\hline Subcell & Model $(\Omega / \square)$ & Nominal $(\Omega / \square)$ \\
\hline GaInP & 1850 & 717 \\
GaAs & 100 & 151 \\
\hline
\end{tabular}


thicknesses of the layers are usually very well controlled, the dopant density specially at high levels - and the carrier mobility can be subjected to large uncertainties. For example, the electron mobility in GaInP with a doping level of $10^{18} \mathrm{~cm}^{-3}$ ranges from 500 to $1000 \mathrm{~cm}^{2} / \mathrm{V} \mathrm{s}$ [20].

As described above, $\mathrm{I}_{01}$ and $\mathrm{I}_{02}$ are obtained from a fitting of the EL-based IV curve. Since this is, in itself, influenced by the sheet resistance and the lateral currents as discussed in Section 3.1, it is reasonable to consider them unreliable. Moreover, ideally, we would like to obtain the solar cell properties just from one, contactless set of measurements: the PL-based IV. A fitting of the three parameters, the two saturation currents and the sheet resistance, is possible as long as the experimental data covers a large enough current injection range. Looking at the curves in Figure 5, it can be seen that this type of absolute fitting is not possible in our case. While the lower injection regime, dominated by lateral transport, is clear in both cases, the higher injection one with no lateral currents is barely seen, specially in the case of GaAs that have small sheet resistance.

\section{Conclusions}

In this work we have presented a method for modelling PL- and EL-based IV curves when the carrier injection, either optical or electrical, is not homogeneous on the whole device. The theoretical formalism models the solar cell as a 3D network of electrical circuits incorporating diodes to represent the recombination mechanisms in the solar cells and resistors to represent the lateral transport processes.

The modelled IV curves support the interpretation that the presence of a finite sheet resistance makes both curves different that the true IV curve of the device, dependent just on the recombination mechanisms. For low sheet resistance, the PL-based IV will tend to overestimate the current. For high sheet resistance, the EL-based IV will overestimate the current.

We have used the method to fit experimental data in the case of GaAs and GaInP solar cells. The model correctly reproduced the experimental data, producing sheet resistance values, taken as a fit parameter, close to the estimated values, based on the nominal structure of the samples.

In summary, these modelling process is a very valuable complement to the luminescence-based IV characterisation methods, being of special interests its potential application to each subcell in MJ devices, where information of sheet resistances and recombination currents are not easily accessible. 


\section{Acknowledgments}

We acknowledge the financial support from the SolCell project through the EMRP Researcher Grant Contract NO. ENG51-REG3. EMRP is jointly funded by the EMRP participating countries within EURAMET and the European Union.

\section{References}

[1] Trupke, T., Bardos, R. A., Schubert, M. C., and Warta, W. (2006). Photoluminescence imaging of silicon wafers. Appl. Phys. Lett. 89, 044107-044114.

[2] Trupke, T., Bardos, R. A., Abbott, M. D., and Cotter, J. E. (2005). Suns- photoluminescence: Contactless determination of current-voltage characteristics of silicon wafers. Appl. Phys. Lett. 87:093503.

[3] Peloso, M. P., Sern Lew, J., Trupke, T., Peters, M., Utama, R., and Aberle, A. G. (2011). Evaluating the electrical properties of silicon wafer solar cells using hyperspectral imaging of luminescence. Appl. Phys. Lett. 99:221915.

[4] Fuyuki, T., Kondo, H., Yamazaki, T., Takahashi, Y., and Uraoka, Y. (2005). Photographic surveying of minority carrier diffusion length in polycrystalline silicon solar cells by electroluminescence. Appl. Phys. Lett. 86:262108.

[5] Kirchartz, T., Helbig, A., Reetz, W., Reuter, M., Werner, J. H., and Rau, U. (2009). Reciprocity between electroluminescence and quantum efficiency used for the characterization of silicon solar cells. Prog. Photovolt Res. Appl. 17, 394-402.

[6] Kirchartz, T., Rau, U., Hermle, M., Bett, A. W., Helbig, A., and Werner, J. H. (2008). Internal voltages in GaInP/GaInAs/Ge multijunction solar cells determined by electroluminescence measurements. Appl. Phys. Lett. 92:123502.

[7] Nesswetter, H., Dyck, W., Lugli, P., Bett, A. W., and Zimmermann, C. G. (2013). Luminescence based series resistance mapping of III-V multijunction solar cells, J. Appl. Phys. 114:194510.

[8] Nesswetter, H., Lugli, P., Bett, A. W., and Zimmermann, C. G. (2013). Electroluminescence and Photoluminescence Characterization of Multijunction Solar Cells. IEEE Photovoltaics J. 3, 353-358.

[9] Roensch, S., Hoheisel, R., Dimroth, F., and Bett, A. W., Subcell I-V (2011). characteristic analysis of GaInP/GaInAs/Ge solar cells using electroluminescence measurements, Appl. Phys. Lett. 98:251113. 
[10] Shu, G.-W., Ou, N.-N., Hsueh, P.-Y., Lin, T.-N., Wang, J.-S., Shen, J.L., et al. (2013). Measuring Photovoltages of III-V Multijunction Solar Cells by Electroluminescence Imaging. Appl. Phys. Express 6:102302.

[11] Steiner, M. A., Geisz, J. F., Moriarty, T. E., France, R. M., McMahon, W. E., Olson, J. M., Kurtz, S. R., and Friedman, D. J. (2013). Measuring IV Curves and Subcell Photocurrents in the Presence of Luminescent Coupling, IEEE J. Photovoltaics 3, 879-887.

[12] Alonso-Alvarez, D., and Ekins-Daukes, N., (2016). PhotoluminescenceBased Current-Voltage Characterisation of Individual Subcells In MultiJunction Devices. IEEE J. Photovolt. 6, 1004-1011.

[13] Steiner, M., Philipps, S. P., Hermle, M., Bett, A. W., and Dimroth, F. (2010). Validated front contact grid simulation for GaAs solar cells under concentrated sunlight. Prog. Photovolt. Res. Appl. 19, 73-83.

[14] Nishioka, K., Takamoto, T., Agui, T., Kaneiwa, M., Uraoka, Y., and Fuyuki, T. (2004). Evaluation of InGaP/InGaAs/Ge Triple-Junction Solar Cell under Concentrated Light by Simulation Program with Integrated Circuit Emphasis. Jpn. J. Appl. Phys. 43, 882-889.

[15] Steiner, M., Guter, W., Peharz, G., Philipps, S. P., Dimroth, F., and Bett, A. W. (2011). A validated SPICE network simulation study on improving tunnel diodes by introducing lateral conduction layers. Prog. Photovolt. Res. Appl. 20, 274-283.

[16] Paire, M., Lombez, L., J.-F. Guillemoles, and Lincot, D. (2011). Measuring sheet resistance of CIGS solar cell's window layer by spatially resolved electroluminescence imaging. Thin. Solid Films 519, 74937496.

[17] Jürgens, T., Gütay, L., and Bauer, G. H. (2006). Photoluminescence, open circuit voltage, and photocurrents in $\mathrm{Cu}(\mathrm{In}, \mathrm{Ga}) \mathrm{Se}_{2}$ solar cells with lateral submicron resolution. Thin Solid Films 511, 678-683.

[18] Pieters, B. E. (2011). Spatial modeling of thin-film solar modules using the network simulation method and spice. IEEE J. Photovolt. 1, 93-98.

[19] NGSPICE. (2014). Available at http://ngspice.sourceforge.net

[20] Sotoodeh, M., Khalid, A. H., and Rezazadeh, A. A. (2000). Empirical lowfield mobility model for III-V compounds applicable in device simulation codes. J. Appl. Phys. 87:2890. 


\section{Biographies}

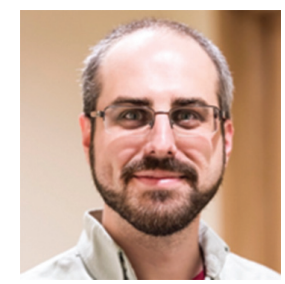

D. Alonso-Álvarez is specialised on the epitaxial growth of III-V semiconductor nanostructures, high efficiency photovoltaic concepts, spectroscopic characterisation techniques and semiconductor device simulation and modelling. Diego got the Ph.D. in 2011 at the Instituto de Microelectrónica de Madrid (Spain), working on strain balanced epitaxial stacks of InAs/GaAs quantum dots and posts for optoelectronic applications. After finishing, he moved to Edinburgh (UK) as research associate at Heriot Watt University, to work on spectral conversion for thin film PV modules. He joined the Quantum Photovoltaic group at Imperial College London as a Marie-Curie post-doctoral researcher in January 2013 to work on the photonic optimization of multi quantum well solar cells, wining a Research Excellence Grant from the EURAMET in 2015 to implement fast, contactless characterisation methods for high efficiency photovoltaics.

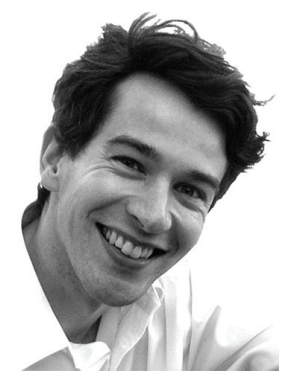

N. Ekins-Daukes is a senior lecturer in Physics department at Imperial College London. His research aims to fundamentally increase the efficiency of photovoltaic solar cells. Specifically, his interests include III-V multi-junction solar cells, intermediate band and hot carrier solar cells. Previously, Ned worked in Australia as a lecturer at the School of Physics at the University of 
Sydney in Australia and as a visiting research fellow at the ARC Photovoltaics Centre of Excellence, UNSW. Prior to that he was a JSPS research fellow at the Toyota Technological Institute, Japan. Ned holds a Ph.D. and MSc from Imperial College and MSci in Physics \& Electronics from the University of St Andrews. 
